\title{
A SmartDisability Framework: enhancing user interaction
}

\author{
Paul Whittington, Huseyin Dogan \\ Faculty of Science and Technology, Bournemouth University \\ Fern Barrow, Talbot Campus, Poole, BH12 5BB, UK \\ \{paul.whittington, hdogan\}@bournemouth.ac.uk
}

\begin{abstract}
This paper introduces the SmartDisability Framework to consider mappings between disability type, Range of Movement and interaction mediums to produce technology and task recommendations to enhance user interaction. The SmartDisability conceptual model (based on the familiar disability symbol) and extracts from the initial development stage of the Framework are presented. The Framework has been populated through the knowledge obtained from state of the art literature reviews of disability classification, Range of Movement, interaction mediums, 'off-the-shelf' technologies and tasks. The Framework was augmented by requirements elicitation results and a described usability evaluation involving a simulation of the SmartATRS smartphone system to control the Automated Transport and Retrieval System (ATRS). ATRS is a technically-advanced system that enables a powered wheelchair (powerchair) to autonomously dock onto a platform lift of a vehicle using an automated tailgate and a motorised driver's seat. The usability of touch and head-based interaction methods were measured using System Usability Scale (SUS) and NASA Task Load Index (NASA TLX) and demonstrated that fingers were more usable interaction method, as head tracking required a full range of neck movement. This SmartDisability Framework is anticipated to be validated through focus groups utilising fictional personas that involve experts from the domains of healthcare, computing and occupational therapy. The framework will be routed to exploitation through the development of a smartphone or web-based application.
\end{abstract}

Assistive Technology. Controlled Usability Evaluation. Framework. NASA Task Load Index. People with Disability. Pervasive Computing. System Usability Scale. User Interaction.

\section{INTRODUCTION}

Approximately 500 million people worldwide have disabilities that affect their interaction with society and the environment (Cofré et al. 2012). Smart technology has proliferated over recent years (Suarez-Tangil et al. 2013) due to the popularity of smartphones and other smart devices (e.g. SmartTVs, tablets and wearable devices) that have the potential to improve quality of life, particularly for people with disability. A user survey conducted by Ari and Inan (2010) highlighted that people with disabilities are often unaware of how technology can provide assistance with daily tasks and improve their quality of life. This indicates the importance of promoting assistive technology solutions, which can be achieved through the proposed SmartDisability Framework.

Previously performed research included a requirements elicitation survey (Whittington et al. $2015 b)$ to establish the types of tasks that were difficult for powered wheelchair (powerchair) users to perform and the technologies that could provide assistance with daily tasks. The smartphone system, SmartATRS (Whittington et al. 2015a), is an example of one such technology to increase the usability of the Automated Transport and Retrieval System (ATRS). ATRS is a technically-advanced system that uses robotics and Light Detection and Ranging (LiDAR) technology to create a reliable, robust means for a wheelchair user to autonomously dock a powerchair onto a platform lift without the need of an assistant (Gao et al. 2008). The rationale behind creating a SmartATRS smartphone interface was to replace the existing very small ATRS keyfobs (similar to those used to control automated gates), which were difficult to operate for people with dexterity impairment and could be easily dropped. SmartATRS was also viewed as a System of Systems (SoS) as it consisted of a number of independently operable constituent systems (Whittington and Dogan 2015).

Feasibility trials have been performed by integrating currently available technologies into SmartATRS and assessing their suitability for people with disabilities. The trials consisted of monitoring brain activity using an electroencephalograph (EEG), tracking facial features through Tracking Learning Detection, applying iOS Switch Control to track the head and 
interacting with a head mounted display. Head tracking proved to have the greatest potential and therefore, a Controlled Usability Evaluation was conducted to assess the usability compared to a standard touch-based interface. The usability evaluation revealed that Range of Movement (ROM) was the determinant as to whether people with disability could operate the technology.

All the research performed to-date has contributed to a resulting SmartDisability Framework which considers the mappings between disability types, ROM, interaction mediums, technologies and tasks. It is a recommendation system that uses physical impairments and ROM characteristics to suggest technologies to assist people with disability to perform daily tasks. The framework therefore, will enable disability to become 'smart', as people with disability can become aware of how technology can improve their quality of life.

\section{STATE OF THE ART}

\subsection{Disability Types}

There are a number of disabilities that exist worldwide as human beings are susceptible to diminishing health and the possibility of developing a disability at any point during life (Kostanjsek 2011). Various frameworks have been developed to categorise disability, including Nagi's model in the 1950s to distribute welfare and economic aid (Nagi 1965), a conceptual model of the Fundamental Principles of Disability (Union of the Physically Impaired Against Segregation 1976), the International Classification of Impairment, Disability and Handicap (ICIDH) (World Health Organization 1980 ) and the model by the National Center for Medical Rehabilitation Research of Bethesda's (NCMRR) for rehabilitation through adapted living environments (US Department of Health and Human Services 2006).

The International Classification of Functioning, Disability and Health (ICF) Framework (World Health Organization 2001) by the World Health Organisation (WHO) is the current international standard for disability classification (Cowan et al. 2012) and was a revision of the original ICIDH recognised by 191 countries (World Health Organization 1980). The framework rationale stated that disability should not characterise individuals, but be a complex interaction method between a person's health conditions and the environment (Kostanjsek 2011). As an addition to the ICF Framework, the WHO subsequently produced the ICF-CY Framework for children and youths (World Health Organization 2007). Research conducted to analyse the relationship between ICF, the Downton Scale and impairment types (Andrews 2014) has mapped disabilities into three categories; 'Motor
Control', 'Senses' and 'Cognitive Ability' each with resulting impairments, such as 'increased/reduced sensation', 'weakness/paralysis/muscle wasting' and 'visual' and examples of physical disabilities, e.g. acquired brain injury, cerebral palsy and muscular dystrophy. The ICF and the classification system provided the impairment types and disabilities for the Disability element of the SmartDisability Framework.

\subsection{Range of Movement (ROM)}

ROM is a measure of movement about the axis of a joint that a person can produce using their own strength (Keilhofner 2006). The ROM of the user was seen as a greater indicator of whether a technology would be suitable rather than a disability type, due to the head tracking technology in the performed user evaluation requiring a full level of ROM. It is accurately measured using a goniometer between 0 to 360 degrees and for the purposes for the SmartDisability Framework, ROM was classified as a Boolean parameter, i.e. the user could or could not produce the movement. There are defined terms relating to impairments that adversely affect ROM, e.g. a limited movement of any joint is known as reduced normal Range of Motion of the joint (O'Connell 2012) whereas, a specific impairment (e.g. finger dexterity) is the inability to manipulate objects using fingers and can be a contraindication of disabilities such as Cerebral Palsy (Georgia Tech Research Institute 2007). Joint hypermobility (also known as 'double jointed') is when some or all of a person's joints have an unusually large ROM (Department of Health 2016). ROM can also be affected by susceptibility of joints being forced out of position, i.e. dislocation (U.S. National Library of Medicine).

\subsection{Impairments}

Impairments can be classified as congenital (i.e. from birth) or acquired (University of WisconsinExtension 2010) (e.g. a traumatic injury or medical condition). Examples of congenital defects are contractures, dystonia and visual impairments. Contractures develop when normally elastic tissues are replaced by inelastic fibre-like tissues preventing normal ROM (U.S. National Library of Medicine 2016). Dystonia is involuntary, variable movement noticeable when the person attempts to move (Cerebral Palsy Alliance 2016). Visual impairments are classed as any form of vision loss, irrespective of whether the person has partial or full loss (The Nemours Foundation 2016). Acquired impairments include paraplegia, cataracts and hemiparesis Paraplegia is the complete or incomplete paralysis affecting the legs and the trunk, but not the arms, as a result of a spinal cord injury where the level of injury in the spine determines the extent to which the trunk is affected 
(Spinal Injury Network 2016). Cataracts can develop at any point in life and are cloudy patches on the lens of the eye causing blurred or misty vision (Department of Health 2016). Hemiparesis is weakness on one side of the body where the affected side has reduced muscle strength and commonly occurs from brain haemorrhage (National Stroke Association 2016).

\subsection{Technologies}

ATRS uses a laser guidance system comprising of a compact LiDAR device coupled with a robotics unit, which is fitted to the powerchair for locating the exact position of the lift and to drive the powerchair onto the lift. Using a joystick attached to the driver's seat, the user manoeuvres the powerchair to the rear of the vehicle until the LiDAR unit is able to see two highly reflective fiducials fitted to the lift. From then on, the docking of the powerchair is completed autonomously. The autonomous control area is shown in Figure 2. If the powerchair drives outside this area, it will stop instantly and requires manual control via the joystick to return the chair into the autonomous control area.

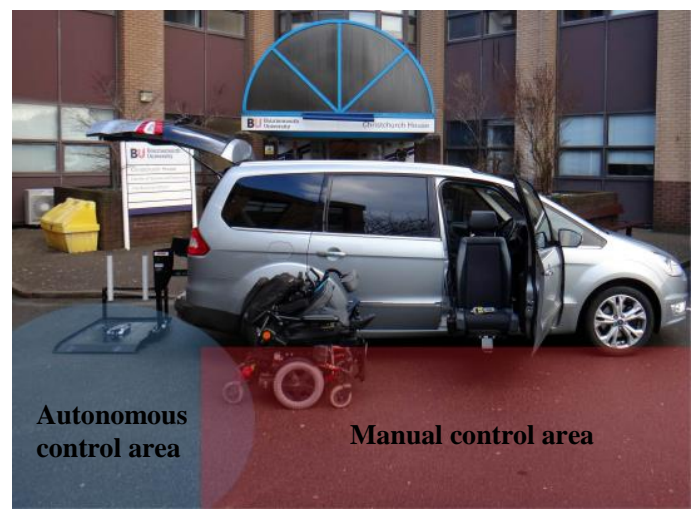

Figure 2: ATRS Operating Zones

ATRS requires the vehicle to be installed with a seat that rotates and exits the vehicle through the driver's door (to enable easy transfer between the powerchair and the driver's seat), an automated tailgate and a lift fitted in the rear boot space. Although there is an autonomous aspect to ATRS, it is seen as an interactive system that requires user interaction to operate the seat, tailgate and lift. Wireless keyfobs and handheld pendants were the only method of interaction in the standard ATRS and this presented a limitation in terms of Human Computer Interaction $(\mathrm{HCl})$

\section{METHODOLOGY}

The smartphone system, SmartATRS, was developed to eliminate the small keyfobs which were difficult to operate for people with dexterity impairment and could be easily dropped.
SmartATRS can be operated by touch, joystick or head tracking interaction methods, providing a significant improvement in the usability of the system. To integrate the system architecture with the standard ATRS, a relay board was used to interface between the ATRS components and the JavaScript. The relay board contained an embedded web server storing the HyperText Markup Language (HTML) and JavaScript GUls as webpages. Ethernet was used to connect the web server to a Wi-Fi router located in the rear of the vehicle. A smartphone communicated with the WiFi router over a secured Wi-Fi network and the GUI could be loaded by entering the Uniform Resource Locator (URL) of the webpage. Joystick control of SmartATRS was achieved using iPortal (Dynamic Controls 2016) that communicated with a smartphone via Bluetooth and also enabled the device to be securely mounted onto the arm of the powerchair, making the system easier to use.

A Controlled Usability Evaluation was conducted to compare head tracking with the existing touchbased interaction method of SmartATRS (using fingers) thereby, determining whether the usability was enhanced by technology insertion. Through observations and the application of the System Usability Scale (SUS) (Brook, 1996), and NASA Task Load Index (TLX) (National Aeronautics and Space Administration, 1996), the evaluation provided a means to determine which interaction method was the most suitable for specific disabilities.

\subsection{Evaluation set-up}

As the installation of SmartATRS was required for daily use, a simulation was used to eliminate risks to the system. An advantage of using a simulation was that the evaluation could be performed in an indoor environment without potential outdoor difficulties.

The SmartATRS simulation consisted of a relay board with an embedded web server (identical to the relay board located in the vehicle), smartphone, and a Windows laptop. The web server on the relay board was connected to a Wireless LAN (WLAN) module, so that a smartphone was able to connect to the relay board wirelessly. The same user interface for SmartATRS existed for the simulation and the relays were operated by the JavaScript. However, the relays were not connected to any functions. A Windows laptop was also connected to the relay board wirelessly to execute a separate piece of JavaScript code that was continuously monitoring the state of the relays. Video clips were displayed depending on the currently operating relay. As the files were too large to be stored on the webserver, the laptop stored video clips locally as MPEG-4 files of the following functions; seat driving into and out of the vehicle, automated tailgate 
closing and opening, lift driving into and out of the vehicle. All six video clips were displayed on the laptop screen. When a relay operated, the appropriate video played and stopped either when the function completed or when the relay switched off prior to completion. In this case, the video paused and resumed once the relay was switched on. It was not possible for opposite motion videos (i.e. Seat $\mathrm{In}$ and Seat Out) to be played simultaneously, as this is not possible in a real system. Therefore, the video paused the opposite motion video.

A separate user interface was created for the SmartATRS simulation. The JavaScript code was different to the standard SmartATRS interface as it read the relays status and did not control the relays. The same stylesheets were used as in standard interface to maintain consistency between the smartphone and laptop displays.

\subsection{Participants and Procedure}

Three organisations were approached to establish a niche user group of 17 participants for the user evaluation. They were a mixture of genders who also had varying disabilities requiring the use of a powerchair or wheelchair (such as Cerebral Palsy, Duchenne's Muscular Dystrophy and Ataxia Telangiectasia) with either dexterity and/or speech impairment. The participants thereby became a representative sample to accurately assess the usability of the interaction methods. As the evaluation was contacted with a user group classed as vulnerable, ethical approval was sought from the Bournemouth University ethics panel prior to conduction. Informed consent was obtained from all participants and a participant information sheet was given to each participant prior to the evaluation. The feedback obtained from the participants could not be obtained from any other type of user group. The participants used the tablet to control the SmartATRS simulation. The usability of the interaction methods were assessed by observing whether the video clip playing on the laptop corresponded to the function that the participant intended to activate. If the video clip did not correspond, an error was made by the participant during the selection process.

\subsection{SUS and NASA TLX}

The usability was also measured by the participants completing SUS and NASA TLX questionnaires provided after the evaluation. The questionnaires were simplified as the participants would not have had the cognitive ability to complete the standard formats. The SUS questionnaire contained 10 statements on a five-point scale from 'No' to 'Yes' (rather than from 'Strongly Disagree' to 'Strongly Agree'). The statements were also simplified and included: "I felt very happy using my fingers to press the buttons on the iPad" and "I thought that using head movements to work the iPad was easy". The NASA TLX questionnaire was modified by naming the extremes of the scale more simplistically (e.g. "Not Hard" and "Very Hard") and simplifying the questions to "How hard was it to move your fingers to press the buttons", for example.

SUS was selected as a usability measurement, as each participant was able to provide a single score in relation to each question (Bangor et al. 2008), enabling SUS scores to be calculated for both interaction methods. The NASA TLX questionnaire concerned the workload experienced during the tasks by measuring the Physical, Mental, Temporal, Performance, Effort and Frustration demands. It is a well-established method of analysing a user's workload and is a quick and easy method of estimating workload that can be implemented with a minimal amount of training (Stanton et al. 2005).

Instead of NASA TLX, the Subjective Workload Dominance Technique (SWORD) could have been implemented to measure the workload experienced. SWORD is not as widely used as NASA TLX (Stanton et al. 2005) with the main difference being that SWORD rates the workload dominance of one task against another. Therefore, SWORD only provides a rating for which tasks create greater workload than others and not a rating of the participant's workload. This would not have been suitable for SmartATRS, as the differences between the interaction methods needed to be measured rather than the differences in domination between the tasks (Human Factors Design and Evaluation Methods Review).

\section{RESULTS}

\subsection{SUS}

The Adjective Rating Scale (Bangor et al., 2009) was used to interpret the SUS scores, with fingers achieving a score of 75.7 (Good Usability) and head tracking achieving 36.7 (Poor Usability). This clearly highlights that fingers is the most usable; with most participants finding head tracking challenging due to the ROM required to operate Switch Control.

A second important result identified the safety of the emergency stop function with each interaction method. The function involved stopping the lift using the Emergency Stop button on the interface a4s soon as the command "Stop!" was given. This task was video recorded so that the time between the command and the emergency stop being selected could be measured. The results revealed a standard deviation of 4 seconds for the fingers, compared to 14 seconds for head tracking. The 
average stopping times were 4 seconds and 16 seconds respectively. The dramatically increased stop times for head tracking were due to the time taken to navigate to the Emergency Stop button using Switch Control, indicating that using the head is more unpredictable than fingers.

\subsection{NASA TLX}

The box plot comparisons in Figure 4 illustrate the differences in the workload experienced when using fingers and head tracking.

From the minimum, lower quartile, median, upper quartile and maximum values, it is evident that 'fingers' showed lower Mental and Temporal demands. Thus, proving that head tracking was more mentally and stressful to complete efficiently. A second important observation was the considerably higher Physical Demand for head tracking resulting in participants either not being able to sufficiently use Switch Control at all or finding it extremely challenging. This observation was seen in $65 \%$ of the participants and led to consideration being given as to why. Switch Control relies on the user having a full range of neck movement i.e. $80^{\circ}$ to the left or right to initiate the interaction and any reduction prevents head movements being detected sufficiently. The remaining $35 \%$ of participants experienced low workload levels when using head tracking due to having full range of neck movement. The limitations of head tracking are also reflected by the increased Effort and Frustration levels compared to 'fingers' Overall the box plots were fairly conclusive that in this particular instance, the use of fingers was more effective than head tracking.
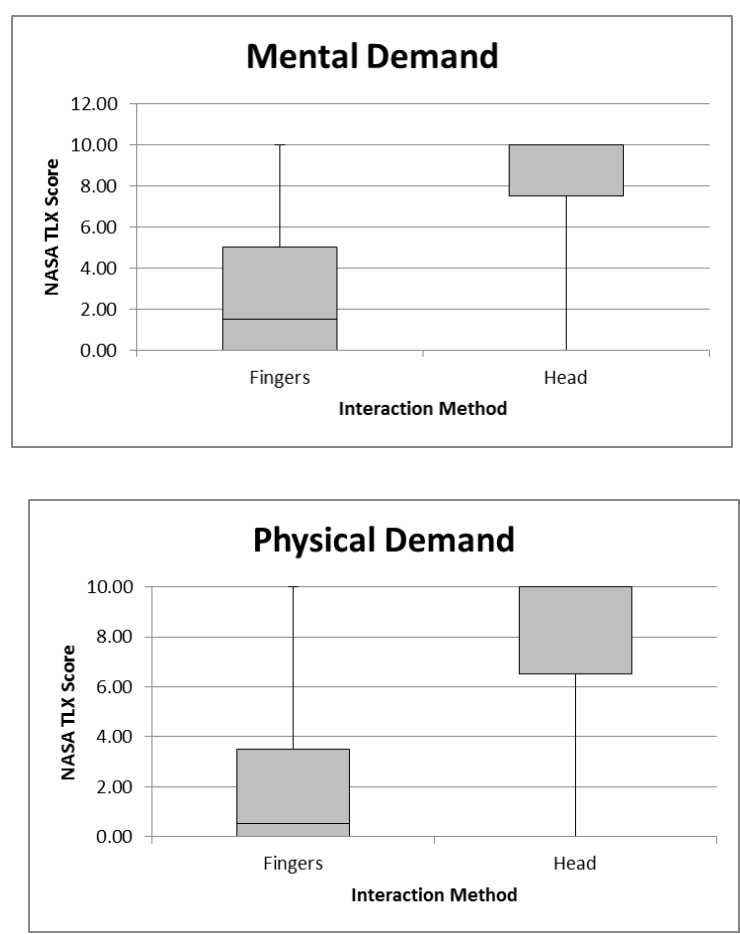

Figure 3: Comparing Mental and Physical Demand experienced
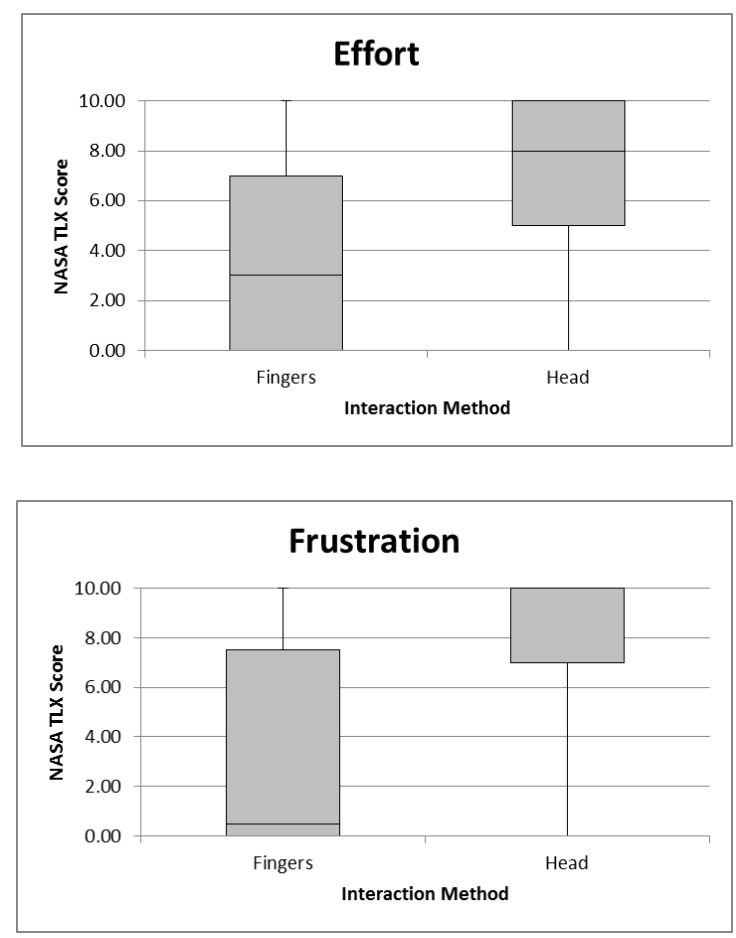

Figure 4: Comparing Effort and Frustration experienced

This comparison between fingers and head interaction methods contributed to the development and population of a SmartDisability Framework to provide technology recommendations based on the knowledge obtained from conducted usability evaluations and state of the art literature reviews. This framework considers the mappings between disability type, ROM and interaction mediums to produce technology and task recommendations.

\subsection{SmartDisability Framework}

The SmartDisability Framework has been established for the healthcare and assistive technology industries and considers how technology can support people with disability and addresses the concept of not having a 'single technology solution to suit all disabilities'. It is currently awaiting validation and consists of seven elements; Disabilities, Impairments, Range of Movements (ROM), Movement Characteristics, Interaction Mediums, Technologies and Tasks, interlinking aspects of $\mathrm{HCl}$. The relationship is illustrated in a linear conceptual model based on the familiar disability symbol, with Disabilities being the input. The purpose of the Impairments element was to condense the large number of disability types into generic forms of impairment. The role of the ROM element is to identify how the 
impairments affect the user's range of movement. It was necessary to consider ROM, as this is the determinant as to whether a user can operate an interactive medium, as identified by the usability evaluation. The results of Controlled Usability Evaluation identified the significance of ROM to determine whether certain users can operate technology.

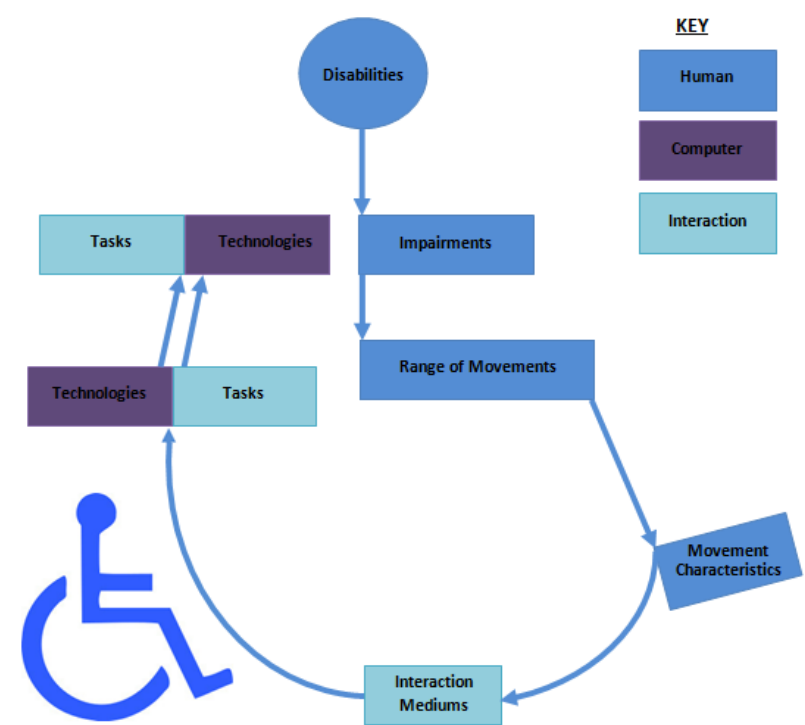

Figure 5: Conceptual model of the SmartDisability Framework

The initial framework is in a spreadsheet format containing individual worksheets for each element with images and references provided for information purposes. It will be converted into a database to ensure data integrity between the elements, allowing an application to be developed for exploitation purposes.

\subsubsection{Disabilities}

The Disabilities table (Figure 6) identifies the physical impairments associated with specific disability types such as an Acquired Brain Injury and Cerebral Palsy, to filter the range of disabilities into generic impairment types. The checkmarks infer that the impairment is a contraindication of a disability, colour-coded depending on the literature source.

\begin{tabular}{|c|c|c|}
\hline \multirow{2}{*}{ Impairments } & \multicolumn{2}{|c|}{$\left.\right|^{2}$} \\
\hline & \multicolumn{2}{|c|}{\begin{tabular}{|l|l|l} 
Acquired Brain Injury & Cerebral Palsy \\
\end{tabular}} \\
\hline sims & & \\
\hline Limited neck movement & $\checkmark$ & $\checkmark$ \\
\hline Limited shoulder movement & $\checkmark$ & $\checkmark$ \\
\hline Limited elbow movement & $\checkmark$ & $\checkmark$ \\
\hline Limited vrist movement & $\checkmark$ & $\checkmark$ \\
\hline Limited finger dexterity & $\checkmark$ & $\checkmark$ \\
\hline Limited ankle movement & $\checkmark$ & $\checkmark$ \\
\hline \multicolumn{3}{|l|}{\begin{tabular}{|l|} 
Joint hypermobility \\
\end{tabular}} \\
\hline \multicolumn{3}{|l|}{\begin{tabular}{|l|} 
Joint dislocation \\
\end{tabular}} \\
\hline Scoliosis & & $\checkmark$ \\
\hline \multicolumn{3}{|l|}{\begin{tabular}{|l} 
Menoziss \\
\end{tabular}} \\
\hline Contractures & $\checkmark$ & $\checkmark$ \\
\hline Dyskenesia & & $\checkmark$ \\
\hline \multicolumn{3}{|l|}{ Atrophy } \\
\hline Paraplegia & $\checkmark$ & $\checkmark$ \\
\hline Quadraplegia / tetraplegia & $\checkmark$ & $\checkmark$ \\
\hline Hemiparesis & $\checkmark$ & $\checkmark$ \\
\hline \multicolumn{3}{|l|}{ 经的 } \\
\hline Visual & $\checkmark$ & $\checkmark$ \\
\hline Cataracts & & \\
\hline
\end{tabular}

Figure 6: An extract of the Disabilities table The impairment types are categorised depending on the affected body parts; 'Joints', 'Muscles', 'Vision' and 'Sensory'. The input to the table is the disability type of the user and this is used to produce a list of affected body parts, leading to input to the ROM table.

\subsubsection{ROM}

The ROM table (Figure 7Figure ) considers how impairment types restrict the ROM of an individual, categorised into associated ROM types.

\begin{tabular}{|l|l|l|l|}
\hline \multicolumn{2}{|c|}{ Resulting Impairments } & \multicolumn{3}{c|}{ Associated ROM } \\
\hline \multicolumn{1}{|c|}{ Joints } & \multicolumn{2}{c|}{ Neck } & \multicolumn{2}{l|}{ Shoulder } & Elbow \\
\hline Limited neck movement & $\checkmark$ & & \\
\hline Limited shoulder movement & & $\checkmark$ & \\
\hline Limited elbow movement & & & $\checkmark$ \\
\hline Limited wrist movement & & & \\
\hline Limited finger dexterity & & & \\
\hline Limited ankle movement & & & \\
\hline Joint hypermobility & & $\checkmark$ & $\checkmark$ \\
\hline Joint dislocation & $\checkmark$ & $\checkmark$ & $\checkmark$ \\
\hline Twisted spine & $\checkmark$ & $\checkmark$ & \\
\hline & \multicolumn{3}{|l|}{} \\
\hline Contractures & $\checkmark$ & $\checkmark$ & $\checkmark$ \\
\hline Dyskenesia & $\checkmark$ & $\checkmark$ & $\checkmark$ \\
\hline Atrophy & $\checkmark$ & $\checkmark$ & $\checkmark$ \\
\hline Paraplegia & $\checkmark$ & $\checkmark$ & \\
\hline
\end{tabular}

Figure 7: An extract of the ROM table

The ROM table forms an input to the ROM Characteristics table, where depending on which type of ROM is affected by the individuals' impairment, the appropriate information is obtained.

\subsubsection{ROM Characteristics}

The measurable features of each ROM type are identified in the ROM Characteristics table (Figure 8Figure ) and have a number of characteristics that determine how the ROM is affected by the impairments. The table uses Boolean statements to determine whether the user can perform each movement and is the input to the Interaction 
Mediums table, enabling suitable interaction mediums to be recommended.

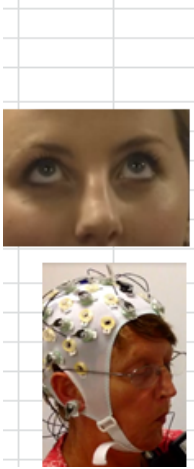

\begin{tabular}{|c|c|c|c|}
\hline \multirow[t]{2}{*}{ ROM Characteristic } & \multicolumn{3}{|c|}{ Measurement Type } \\
\hline & Max Degrees & Boolean & Max Percentage \\
\hline \multicolumn{4}{|l|}{ Eye ${ }^{[2]}$} \\
\hline Gaze up & & $\mathrm{Y} / \mathrm{N}$ & \\
\hline Gaze down & & $\mathrm{Y} / \mathrm{N}$ & \\
\hline Gaze left & & $\mathrm{Y} / \mathrm{N}$ & \\
\hline Gaze right & & $\mathrm{Y} / \mathrm{N}$ & \\
\hline Blinking & & $\mathrm{Y} / \mathrm{N}$ & \\
\hline \multicolumn{4}{|l|}{ Mouth ${ }^{[4]}$} \\
\hline Suck & & $\mathrm{Y} / \mathrm{N}$ & \\
\hline Blow & & $\mathrm{Y} / \mathrm{N}$ & \\
\hline Bite tongue & & $\mathrm{Y} / \mathrm{N}$ & \\
\hline Move tongue left & & $\mathrm{Y} / \mathrm{N}$ & \\
\hline Move tongue right & & $\mathrm{Y} / \mathrm{N}$ & \\
\hline Smiling & & $\mathrm{Y} / \mathrm{N}$ & \\
\hline
\end{tabular}

Figure 8: An extract of the ROM Characteristics table

\subsubsection{Interaction Mediums}

Figure 9 describes the relationship between different interaction mediums and the required ROM for the interaction between a user and technology. The cells of the table are highlighted where the interaction medium requires a particular ROM characteristic, e.g. an eye-based medium requires a user to gaze up, down, left, right or blink. 'Sip n Puff' is an assistive technology device that requires a user to interact through sucking and blowing (Origin Instruments Corporation 2016). Gesture control enables users to create specific gestures with their hands to interact with devices (Platz and Clothier 2015), but is only suitable for users who have full elbow, wrist and hand ROM. The output of the table is a list of interaction mediums that are suitable for the user and represents the input to the Technologies table with any mediums that require a ROM that the user does not possess, being omitted from the recommendation.

\begin{tabular}{|l|l|l|l|l|l|}
\hline \multicolumn{1}{|c|}{$\begin{array}{c}\text { Associated } \\
\text { Characteristic }\end{array}$} & \multicolumn{5}{c|}{ Interaction Mediums } \\
\hline & $\begin{array}{l}\text { Joystick } \\
{[1]}\end{array}$ & $\begin{array}{l}\text { Voice } \\
\text { [6] }\end{array}$ & Head $^{\left[{ }^{[3]}\right.}$ & Eye ${ }^{[2]}$ & $\begin{array}{l}\text { Sip } \text { n Puff } \\
{[4]}\end{array}$ \\
\hline & & & & & \\
\hline Gaze up & & & & & \\
\hline Gaze down & & & & & \\
\hline Gaze left & & & & & \\
\hline Gaze right & & & & & \\
\hline Blinking & & & & & \\
\hline & & & & & \\
\hline Suck Mouth & & & & & \\
\hline Blow & & & & & \\
\hline Bite tongue & & & & & \\
\hline Move tongue left & & & & & \\
\hline Move tongue right & & & & & \\
\hline Smiling Voice & & & & & \\
\hline \multicolumn{1}{|c|}{ Voicen } & & & & & \\
\hline Speech intelligability & & & & & \\
\hline
\end{tabular}

Figure 9: An extract of the Interaction Mediums table

\subsubsection{Technologies}

The Technologies table (Figure 10) identifies the specific technologies that can be operated through each interaction medium, such as smartphones, tablets and built-in eye tracking.

\begin{tabular}{|c|c|c|c|c|}
\hline \multirow{2}{*}{$\begin{array}{l}\text { Interaction } \\
\text { Mediums }\end{array}$} & \multicolumn{4}{|c|}{ Technology } \\
\hline & \begin{tabular}{|c|} 
Smartphone \\
{$[1]$}
\end{tabular} & Tablet [2] & $\begin{array}{l}\text { Head Mounted } \\
\text { Display [3] }\end{array}$ & $\begin{array}{c}\text { Built-in Eye Tracking } \\
{[4]}\end{array}$ \\
\hline \multicolumn{5}{|l|}{ Joystick } \\
\hline \multicolumn{5}{|l|}{ Voice } \\
\hline \multicolumn{5}{|l|}{ Head } \\
\hline \multicolumn{5}{|l|}{ Eye } \\
\hline \multicolumn{5}{|l|}{ Sip n Puff } \\
\hline \multicolumn{5}{|l|}{ Foot } \\
\hline \multicolumn{5}{|l|}{ Chin } \\
\hline \multicolumn{5}{|l|}{ Fingers } \\
\hline \multicolumn{5}{|l|}{ Brain activity } \\
\hline & 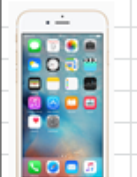 & & [3] & [4] \\
\hline
\end{tabular}

Figure 10: An extract of the Technologies table A Head Mounted Display (HMD) and an EEG are classed as wearable, as the user is required to wear the technology to interact with it. The feasibility trials illustrated that there was an obvious limitation if a person did not possess the required dexterity. Eye tracking can either be a built-in feature of a device (e.g. smartphone) or standalone, which specifically captures the eye movements of the user. Momentary switches enable the user to interact with devices by pressing buttons located in any position, e.g. the headrest or arm of a powerchair. A rear view camera would assist the user with manoeuvring from a live view on a smartphone or tablet attached to the powerchair. Most of the technologies have multiple methods of interaction, e.g. smartphones can be used by either fingers, joystick, head, eye, 'Sip n Puff' and voice, whereas built-in head tracking can only be used with the head. The head tracking experimentation proved that iOS Switch Control was only suitable for users who possessed the necessary neck ROM.

The table provides technology recommendations that are suitable for the ROM of the users and will be the input to the final table of the SmartDisability Framework; the Tasks table.

\subsubsection{Tasks}

The purpose of the Tasks table (Figure 11) is to suggest daily tasks that can be performed with each of the technologies defined in the Technologies table. Most of the technologies can support a variety of tasks whereas, some are specific e.g. a rear view camera can only assist with navigation. 


\begin{tabular}{|c|c|l|l|}
\hline \multirow{2}{*}{ Technology } & \multicolumn{3}{c|}{ Task } \\
\cline { 2 - 4 } & $\begin{array}{l}\text { Navigating } \\
\text { powerchair } \\
{[1]}\end{array}$ & $\begin{array}{l}\text { Operating vehicle } \\
\text { adaptations [1] }\end{array}$ & $\begin{array}{l}\text { Operating cooking } \\
\text { equipment [1][2] }\end{array}$ \\
\hline Smartphone & & & \\
\hline Tablet & & & \\
\hline Head Mounted Display & & & \\
\hline Built-in Eye Tracker & & & \\
\hline Stand-alone Eye Tracker & & & \\
\hline Electroencephalogram & & & \\
\hline Momentary Switches & & & \\
\hline Rear View Camera & & & \\
\hline & & & \\
\hline & & & \\
\hline & & & \\
\hline
\end{tabular}

Figure 11: An extract of the Tasks table The output of the table produces a list of recommended technologies and supported tasks that are suitable for the disability of the user.

\section{DISCUSSION}

A Controlled Usability Evaluation has been described that compared touch and head-based interaction methods. Informative statistics were obtained from the questionnaires, which enabled conclusions to be drawn. This was an effective method of demonstrating that in this particular instance, using fingers was less demanding than using the head, with SUS scores of 75.7 and 36.7 respectively.

The reduced usability of head tracking was reflected by the safety of the Emergency Stop task, where a standard deviation stopping time of 4 seconds was achieved for fingers, compared to 14 seconds for head tracking. This is an unacceptable time for an emergency situation and therefore, proves that head is not a robust means of interaction, as initially anticipated. The importance of robust assistive technologies is acknowledged by Metsis et al. (2008) who recommend that unusual situations must be supported by such technologies to cater for user errors.

NASA TLX analysis identified that head-based interaction generated increased physical, mental and temporal demands as well as greater effort and frustration levels. The difference in physical demands for the two interaction methods was primarily the result of participants who did not possess the required ROM of the neck for iOS Switch Control to recognise the head movement. The findings led to the realisation that disability type is not the determinant as to whether a technology or interaction method would be suitable to a person with disability. The establishment of a SmartDisability Framework aims to address this issue by recommending interaction mediums, technologies and tasks depending on the disability, impairments and ROM of the user, instead of by disability type. Analysing physical disabilities identified common impairments used to characterise the types of ROM that affected disability and formed the basis of the ROM element of the framework. Measurement techniques using Boolean parameters were applied to the ROM Characteristics element to determine the suitable interaction mediums, as each medium was related to a required ROM. Currently available technologies were contained within the Technology element and each had defined supported interaction mediums. The Task element described daily tasks that users wished to perform with the assistance of technology. The relationships between technologies and tasks were established from tasks that were difficult for people with disabilities to perform to investigate whether new technologies could provide an alternative method of performing a task. Developing the SmartDisability Framework was motivated by state of the art reviews concluding that a technology recommendation system for people with disabilities does not current exist. It was also shown by Ari and Inan (2010) that people with disabilities are often unaware of assistive technologies. It is anticipated through using the framework, people with disabilities will become aware of the ways that technology can support their daily lives.

\section{FUTURE WORK}

Further technologies will be assessed using the SmartATRS simulation to determine the suitability of the interaction methods for people with disability. Following a successful feasibility trial, a Controlled Usability Evaluation will be conducted using a HMD (Recon Instruments 2016). Due to the operating system of the HMD, an alternative interface will be developed for SmartATRS. This will consist of reducing the size of the buttons, so that it can be viewed on the display. The SUS and NASA TLX techniques will be similarly applied to the evaluation to enable a comparison in the usability and workloads experienced to be made in comparison with touch-based and head tracking interfaces. The results will further contribute to the SmartDisability Framework.

The framework will be validated through the involvement of domain experts from healthcare, computing and occupational therapy. The healthcare experts will validate the disability types, impairments and ROM sections, computing experts will validate the technology-related aspects and occupational therapy experts will validate the tasks. The purpose of the validation is to ensure that all seven elements of the framework are suitably robust for exploitation to the assistive technology domain. The validation process will take the form of a focus group that will utilise specialist domain knowledge, where each participant will be allocated at least two disabilities, ROM, ROM 
Characteristics, Technologies or Tasks. Fictional personas will also be applied to test the framework consisting of the physical impairments and the ROM of a fictional individual and asked to create technology recommendations for a persona. The results will be further validated to see whether the technology and tasks will be applicable to an individual.

The validated framework will be disseminated to assistive technology industries, as well as to healthcare professionals to suggest technologies that could support the daily lives of their clients with disability. It is anticipated that in the future, framework expansion will be through the creation of additional columns and rows in the tables, as new forms of interaction methods, technologies and tasks are established. To maintain the framework integrity, it will be necessary for any new aspect to be mapped to an associated element.

Dissemination and exploitation of the framework is expected through the development of a smartphone or web-based application. Users would input their impairments, and associated ROM Characteristics to the application and by utilising the contents of the framework, technology recommendations with supported tasks would be suggested.

\section{CONCLUSIONS}

Touch and head-based interaction methods have been evaluated and shown that for SmartATRS, using fingers is more suitable due to head tracking requiring a full ROM of the neck. Both interaction methods met the functionality metric defined by Metsis et al. (2008), stating that "an assistive technology must perform correctly in order to serve its purpose", however, through observation, the SUS and NASA TLX results, it became apparent that ROM is a key determinant of an efficient interaction method. Based on this outcome, the SmartDisability Framework was developed to recommend technology solutions relating to the impairments and ROM of the user. The initial development phase of the framework was the result of a state of the art literature review into disability classification and impairments, ROM types and characteristics, technologies and tasks. The framework considered the wide-range of possible physical disabilities and condensed them into a set of resulting impairments that were used to characterise the affected ROM of a user.

The anticipated SmartDisability application will be used by either people with disability to discover technologies that are available, or by industries and healthcare professionals to assist their clients with disability in their daily lives. Considering the proliferation of smart technology over recent years (Suarez-Tangil et al. 2013), it is expected that the
SmartDisability Framework will enhance user interaction, allowing disability to become 'Smart' and potentially improve quality of life by providing independence.

\section{REFERENCES}

Andrews, R. (2014) Disabling conditions and ICF measures. Cambridge: Addenbrooke's Hospital. Unpublished.

Ari, I. A. and Inan, F. A. (2010). Assistive Technologies for Students with Disabilities: A Survey of Access and Use in Turkish Universities. Turkish Online Journal of Educational Technology, 9, 40-45.

Cerebral Palsy Alliance. (2016) Dyskinetic Cerebral Palsy. https://www.cerebralpalsy.org.au/what-iscerebral-palsy/types-of-cerebral-

palsy/dyskinetic-cerebral-palsy (Retrieved 22 May 2016)

Cofré, J.P., Moraga G., Rusu, C., Mercado, I., Inostroza, R. and Jiménez, C. (2012) Developing a Touchscreen-based Domotic Tool for Users With Motor Disabilities. The 9th International Conference on Information Technology: New Generations, Las Vegas, NV, USA, 16-18 April 2012. 696-701. IEEE Press, New York, NY, USA.

Cowan, R. E., Fregly, B. J., Boninger, M. L., Chan, L., Rodgers, M. M. and Reinkensmeyer, D. J.. (2012). Recent trends in assistive technology for mobility. NeuroEngineering and Rehabilitation, $\dot{9}, 1-8$.

Department of Health. (2016) Age-related cataracts.

http://www.nhs.uk/conditions/Cataracts-agerelated/Pages/Introduction.aspx (Retrieved 22 May 2016).

Department of Health. (2016) Joint Hypermobility. http://www.nhs.uk/conditions/Jointhypermobility/Pages/Introduction.aspx (Retrieved 22 May 2016).

Dynamic Controls. (2016) iPortal $^{\mathrm{TM}}$ Accessibility http://dynamiccontrols.com/en/iportal/iportalaccessibility (Retrieved 22 May 2016).

Fundamental Principles of Disability (1976). Union of the Physically Impaired Against Segregation, London.

Georgia Tech Research Institute. (2007) Dexterity and Mobility Impairment Fact Sheet. http://accessibility.gtri.gatech.edu/assistant/acc_i nfo/factsheet_dexterity_mobility.php (Retrieved 22 May 2016).

Human Factors Design and Evaluation Methods Review.

http://www.hfidtc.com/research/methods/method 
s-reports/phase-1/hf-design-evaluationmethods.pdf.

ICF (1980) International Classification of Impairments, Disabilities and Handicaps: A manual of classification relating to the consequences of diseases, published in accordance with resolution WHA 29.35 of the 29th World Health Assembly. World Health Organization, Geneva.

ICF (2001) International Classification of Functioning, Disability and Health: Short Version: ICF. World Health Organization, Geneva.

ICF-CY (2007) International Classification of Functioning, Disability and Health: Children \& Youth Version: ICF. World Health Organization, Geneva.

ICIDH (1980) World Health Organization, International Classification of Impairment, Disability and Handicap. World Health Organization, Geneva.

Keilhofner G. (2006) Research in Occupational Therapy: Methods of Inquiry for Enhancing Practice. F.A. Davis Company, Philadelphia, PA, USA.

Kostanjsek, N. (2011). Use of The International Classification of Functioning, Disability and Health (ICF) as a conceptual Framework and common language for disability statistics and health information systems. BMC Public Health, 1, 1-6.

Metsis, V., Zhengyi, L., Lei, Y. and Makedon, F. (2008) Towards an Evaluation Framework for Assistive Technology Environments. The 1st International Conference on PErvasive Technologies Related to Assistive Environments, Athens, Greece, 15-19 July 2008. 12 ACM Press, New York, NY.

Nagi, S. Z. (1965). Some conceptual issues in disability and rehabilitation. Sociology and Rehabilitation.

National Stroke Association. (2016) Hemiparesis http://www.stroke.org/we-can-

help/survivors/stroke-recovery/post-strokeconditions/physical/hemiparesis (Retrieved 22 May 2016).

O'Connell, K. (2012) What causes limited range of motion? 20 possible conditions. http://www.healthline.com/symptom/limitedrange-of-motion (Retrieved 22 May 2016).

Origin Instruments Corporation. (2016) Sip and Puff Switch Solutions: Single and Multi-User Offerings. http://www.orin.com/access/sip_puff/ (Retrieved 22 May 2016).

Platz, J. and Clothier, K. (2015) Motion-controlled Servos with Leap Motion \& Raspberry Pi. https://www.pubnub.com/blog/2015-08-19- motion-controlled-servos-with-leap-motionraspberry-pi/ (Retrieved 22 May 2016).

Recon Instruments. (2016) Recon JET: Smarter eyewear. Built by athletes for athletes. http://www.reconinstruments.com/products/jet/ (Retrieved 22 May 2016).

Report to the NACHHD Council (2006). US Department of Health and Human Services, Washington.

Spinal Injury Network. (2016) What is Paraplegia? http://www.spinal-injury.net/paraplegia.htm

(Retrieved 22 May 2016).

Stanton, N. A., Salmon, P. M., Walker, G. H., Baber, C. and Jenkins, D. P. Human Factors Methods: A Practical Guide for Engineering and Design, 2005, 315-320.

Suarez-Tangil, G., Tapiador, J., Peris-Lopez, P., Ribagorda, A. (2013). Evolution, Detection and Analysis of Malware for Smart Devices. Communications Surveys \& Tutorials, 99, 1-27.

The Nemours Foundation. (2016) Visual Impairment

http://kidshealth.org/teen/diseases_conditions/si ght/visual_impairment.html (Retrieved 22 May 2016).

U.S. National Library of Medicine. (2016) Contracture deformity. https://www.nlm.nih.gov/medlineplus/ency/article /003185.htm (Retrieved 22 May 2016).

U.S. National Library of Medicine. (2016) Dislocations.

https://www.nlm.nih.gov/medlineplus/dislocation. html (Retrieved 22 May 2016).

University of Wisconsin-Extension. (2010). Coping with a Disability. fyi.uwex.edu/agrability/files/2010/04/4-Copingwith-a-Disability.ppt (Retrieved 22 May 2016).

Whittington, P. and Dogan, H. (2015) SmartPowerchair: A Pervasive System of Systems. The 10th International Conference on System of System Engineering, San Antonio, TX, USA, 18-20 May 2015. IEEE Press, New York, NY, USA.

Whittington, P., Dogan, H. and Phalp, K. (2015a) Evaluating the Usability of an Automated Transport and Retrieval System. The 5th International Conference on Pervasive and Embedded Computing and Communication Systems, Angers, France, 11-13 February 2015. 59-66. Science and Technology Press, Lisbon, Portugal.

Whittington, P., Dogan, H. and Phalp, K. (2015b) SmartPowerchair: to boldly go where a powerchair has not gone before. Ergonomics \& 
Human Factors 2015, Daventry, UK, 13-16 April

2015. 233-240. CRC Press, London, UK. 